\title{
THE ROLE OF HYDROGEN IN THE FORMATION OF MICROCRISTALLINE SILICON
}

\author{
A.Fontcuberta ${ }^{1}$, J.Bertomeu ${ }^{2}$, P.Roca i Cabarrocas ${ }^{1}$ \\ ${ }^{1}$ LPICM (UMR 7647 CNRS), Ecole Polytechnique, 91128 Palaiseau, France \\ ${ }^{2}$ Departament de Física Aplicada i Òptica, Universitat de Barcelona, Av.Diagonal 647, 08028 \\ Barcelona, Spain
}

\begin{abstract}
The growth mechanisms of microcrystalline films at low temperatures $\left(100-250^{\circ} \mathrm{C}\right)$ by plasma CVD are still a matter of debate. We have shown that $\mu \mathrm{c}-\mathrm{Si}: \mathrm{H}$ formation proceeds through four phases (incubation, nucleation, growth and steady state) and that hydrogen plays a key role in this process, particularly during the incubation phase in which hydrogen modifies the amorphous silicon network and forms a highly porous phase where nucleation takes place. In this study we combine in-situ ellipsometry and dark conductivity measurements with ex-situ high resolution transmission electron microscopy to improve our understanding on microcrystalline silicon formation.
\end{abstract}

\section{INTRODUCTION}

Microcrystalline silicon films $(\mu \mathrm{c}-\mathrm{Si}: \mathrm{H})$ are generally produced by plasma enhanced chemical vapour deposition (PECVD) of silane highly diluted in hydrogen. Since the first report on $\mu c-S i: H$ growth 30 years ago ${ }^{1}$, a large number of models have been proposed to understand the formation of $\mu \mathrm{c}-\mathrm{Si}: \mathrm{H}$ films at low temperatures $\left(50-350^{\circ} \mathrm{C}\right)$ by PECVD. These models can be classified in two categories:

1.Surface models. Within these models one considers only reactions taking place at the surface of the growing film. Among them, the selective etching of the amorphous phase by atomic hydrogen ${ }^{2-3}$ and the enhanced mobility of $\mathrm{SiH}_{3}$ radicals due to the hydrogen coverage of the growing surface ${ }^{4}$. However, none of the surface models does explain the nucleation of silicon crystallites.

2. Growth zone models. Within these models, one considers the chemical reactions taking place on the film surface as well as within a few nanometers-thick subsurface region ${ }^{5-6}$. These chemical reactions involve $\mathrm{Si}-\mathrm{H}$ bond breaking and the recombination of atomic hydrogen to form $\mathrm{H}_{2}$, providing the energy for the rearrangement of the silicon network (chemical annealing), i.e. its crystallisation.

While hydrogen dilution is the most popular technique to produce $\mu \mathrm{c}-\mathrm{Si}: \mathrm{H}$ films, we have used the layer-by-layer (LBL) technique in which the deposition of hydrogenated amorphous silicon (a-Si:H) during a time $\mathrm{T}_{\mathrm{Si}}$ is followed by its exposure to a hydrogen plasma during a time $\mathrm{T}_{\mathrm{H}}$, this cycle being repeated $\mathrm{N}$ times. This allows to simplify the plasma chemistry and to define the transition from a-Si:H to $\mu \mathrm{c}-\mathrm{Si}: \mathrm{H}$ deposition with only two parameters ${ }^{7} \mathrm{~T}_{\mathrm{H}}$ and $\mathrm{T}_{\mathrm{Si}}$. During $\mathrm{T}_{\mathrm{H}}$ atomic hydrogen diffuses through the a-Si:H layer deposited during the time $\mathrm{T}_{\mathrm{Si}}$ and induces its crystallization if $\mathrm{T}_{\mathrm{H}}$ is higher than the time needed by hydrogen to diffuse through the a-Si:H layer deposited during $\mathrm{T}_{\mathrm{Si}}{ }^{8}$. In the case of $\mu \mathrm{c}-\mathrm{Si}: \mathrm{H}$ deposition on glass substrates, the increase of the $\mathrm{T}_{\mathrm{H}}$ while keeping $\mathrm{T}_{\mathrm{Si}}$ constant results in the complete etching of the a-Si:H film deposited during the time $\mathrm{T}_{\mathrm{Si}}$. However, when the LBL process is performed on an a-Si:H substrate the obtention of a $\mu \mathrm{c}-\mathrm{Si}: \mathrm{H}$ film requires a higher $\mathrm{T}_{\mathrm{H}}$ value ${ }^{9}$ and the diffusion of hydrogen results in the formation of a 
porous interface layer which thickness depends on the LBL conditions and the kind of deposited film (intrinsic or doped).

In our previous studies we have combined the LBL technique with in situ ellipsometry analysis of the film properties and validated the optical models with high-resolution transmission electron microscopy (HRTEM) ${ }^{10}$ and secondary ion mass spectrometry measurements ${ }^{11}$. We showed that both surface and growth zone processes are involved in the growth of $\mu \mathrm{c}-\mathrm{Si}: \mathrm{H}^{12-13}$. Moreover, the optical models used to analyse the ellipsometry measurements indicate that the nucleation of crystallites takes place in a highly porous layer below the film surface ${ }^{14}$. In this study, we combine in situ ellipsometry and conductivity measurements with ex-situ HRTEM measurements to confirm that the nucleation takes place in this highly porous layer.

\section{EXPERIMENTAL DETAILS.}

The films were grown at $200{ }^{\circ} \mathrm{C}$ by the LBL technique in a capacitively coupled radio-frequency $(13.56 \mathrm{MHz})$ glow discharge reactor ${ }^{15}$. Table I summarises the deposition conditions used for the growth of the a-Si:H substrate and the $\mu \mathrm{c}-\mathrm{Si}: \mathrm{H}$ films. The ratio $\mathrm{T}_{\mathrm{H}} / \mathrm{T}_{\mathrm{Si}}=6$ corresponds to conditions of no film deposition on Corning glass. A Corning glass provided with two bands of thermally evaporated chromium for in situ dark conductivity measurements was used as a substrate. This substrate was loaded into the reactor for the deposition of a $60 \mathrm{~nm}$ thick a-Si:H film acting as a new substrate for the LBL deposition of $\mu \mathrm{c}-\mathrm{Si}: \mathrm{H}$. Previous to $\mu \mathrm{c}-\mathrm{Si}: \mathrm{H}$ growth, the a-Si:H film was characterised in situ by spectroscopic ellipsometry (SE) and by dark conductivity.

The in situ analysis of the $\mu \mathrm{c}-\mathrm{Si}: \mathrm{H}$ growth was carried out as follows: after some LBL cycles (from 1 to 3), the plasma was switched off and SE and dark conductivity measurements were performed. Then the plasma was turned on to initiate the next series of LBL cycles. Two samples were prepared for HRTEM measurements. In one case (sample 80526A), the LBL process was stopped at 8 cycles so that the film still was in its nucleation phase. For the second sample (80526B), the LBL process was continued until 15 cycles, corresponding to the growth phase of the crystallites was stopped just at the stage of nucleation of the crystallites ${ }^{16}$.

The preparation of the samples for cross sectional HTREM measurements has been previously described $^{10}$.

\section{RESULTS AND DISCUSSION}

\section{IIIa. In situ spectroscopic ellipsometry measurements.}

Figures $1 \mathrm{a}$ and $1 \mathrm{~b}$ show the evolution of the imaginary part of the pseudo-dielectric function $\left\langle\boldsymbol{E}_{2}\right\rangle$ extracted from the spectroscopic ellipsometry measurements performed at different number of LBL cycles. The spectrum at 0 cycles corresponds to the a-Si:H substrate. Taking this spectrum as a reference, we can see that the amplitude of $\left\langle\mathcal{E}_{2}\right\rangle$ decreases gradually during de first nine LBL cycles. The strong decrease in the amplitude of $\left\langle\varepsilon_{2}\right\rangle$ can be explained by the formation of a highly porous and hydrogen-rich surface layer. Moreover, one can notice a change in the shape of the spectra between 6 and 9 cyles. As the number of cycles increases (Figure 1b), the amplitude of $\left\langle\varepsilon_{2}\right\rangle$ follows an opposite evolution, with a sharp increase between 9 and 15 cycles. Therefore, during this stage of the LBL process the porosity of the film decreases. Moreover at 15 cycles one can clearly distinguish the presence of a shoulder near $4.2 \mathrm{eV}$, a signature of the presence of a crystalline phase. 
The spectra shown in Figs. 1 were fitted using the Bruggeman effective medium approximation ${ }^{17}$ (BEMA) to infer the evolution of the composition and thickness of the films. In the optical model used to fit the experimental data we considered the film as a multilayer structure consisting of: i) the a-Si:H substrate, ii) an interface layer between the a-Si:H substrate and de $\mu c-S i: H$ layer, iii) the $\mu \mathrm{c}-\mathrm{Si}: \mathrm{H}$ layer, and iv) a surface roughness. In the BEMA model, the $\mu \mathrm{c}-\mathrm{Si}: \mathrm{H}$ film is described as a mixture of amorphous silicon ${ }^{18}$, polycrystalline silicon ${ }^{19}$ and voids. The a-Si:H layer is described by a mixture of amorphous silicon and voids.

Figure 2 summarises the results of the optical models used to fit the spectra shown in Fig. 1. The ratio $\mathrm{T}_{\mathrm{H}} / \mathrm{T}_{\mathrm{Si}}=6$ corresponds to conditions of no film growth on Corning glass; i.e., the a-Si:H deposited during $\mathrm{T}_{\mathrm{Si}}$ is removed durign $\mathrm{T}_{\mathrm{H}}$. Accordingly, the average deposition rate is very small during the first six cycles. However, the BEMA analysis shows that the porosity of the a-Si:H film increases down to the Corning glass substrate. In the optical model we have considered the a-Si:H film as a combination of two layers: a highly porous on the top and a less perturbed one below. The thickness of the highly perturbed layer with a porosity up to $15 \%$ is $271 \AA$. Therefore, the results of the optical model suggest that the role of hydrogen during the first LBL cycles is to increase the porosity of the a-Si:H film. Actually, this increase in porosity has been shown to correlate with an increase in the hydrogen content measured by SIMS $^{20}$.

The optical model (Fig. 2) shows that the change in the shape of the spectra between six and nine cycles is due to the presence of a crystalline phase. Note that nucleation has taken place in the highly porous surface region. Moreover, the BEMA model predicts an increase in the thickness of the perturbed substrate with a porosity of $15 \%(355 \AA)$. As the number of cycles is further increased up to 15 cycles, the thickness of the crystalline layer reaches $216 \AA$ plus a roughness layer of $35 \AA$. This phase corresponds to the growth of the crystallites within the whole layer. We note also that at 15 cycles the BEMA model still predicts the existence of a porous layer just under the crystalline layer. However, the thickness of this interface layer has decreased with respect to the one at 12 cycles. These results suggest that the a-Si:H substrate is deeply modified during the first 6 LBL cycles and that its effect is reversed once crystallisation starts.

\section{IIIb. In situ dark conductivity measurements.}

To get more insight on the evolution of the film properties we measured in-situ the dark conductivity of the layer. Figure 3 represents the evolution of conductivity for the samples 80526A and $80526 \mathrm{~B}$. The conductivity at the beginning of the LBL process corresponds to that of the a-Si:H film. During the first six LBL cycles we observe a decrease in the conductivity of the film, which can be attributed to the increase of the porosity through the whole a-Si:H (see Fig. 2). Nevertheless, surface band bending effects cannot be excluded, even though one may expect them to be negligible at $200{ }^{\circ} \mathrm{C}$. A bulk effect is further supported by the change of the slope between 6 and 9 cycles, coinciding with the nucleation of the first crystallites. The dark conductivity of the film increased by two orders of magnitude as the thickness and crystalline fraction of the top layer increased.

The changes in the conductivity of the film are thus well correlated with the results of the ellipsometry measurements. The initial decrease in conductivity with the formation of a porous phase, the change in slope with the nucleation phase and the sharp increase of conductivity with a high crystalline fraction, above the percolation threshold. (NO HO ENTENC)

\section{IIIc. HRTEM measurements.}

Two samples were prepared for HRTEM measurements. For sample 80526A, the LBL sequence was stopped during the nucleation phase ( 8 cycles), while for sample $80526 \mathrm{~B}$ the LBL process 
continued until the growth phase of crystallites at 15 cycles. Figure $4 \mathrm{a}$ and $\mathrm{b}$ show the results of high resolution transmission electron microscopy measurements (HRTEM) performed on these samples. First, one can clearly distinguish the presence of a low density region corresponding to the porous layer deduced from the ellipsometry measurements. Moreover, the evolution of this porous layer is also qualitatively in agreement with the results of the SE model. The low density regions are large and disconnected at 8 cycles, while it becomes more continuous and dense at 15 cycles. Moreover, one can see that the nucleation of the first crystallites indeed takes place around 8 cyles and that at 15 cycles the crystallites have grown in size. The thickness of the initial a-Si:H substrate (590 $\AA$ ) and that of the microcrystalline silicon layer $(207 \AA)$ are also shown in Fig 4b. These values are in excellent agreement with those deduced from the optical model. One can notice that, as deduced from ellipsometry model, the a-SiH substrate has been modified over $10 \mathrm{~nm}$. Finally, the HRTEM pictures support the idea that the nucleation takes place in the porous layer. One can see the crystallites to grow up from the porous layer.

In a whole, the above results indicate that hydrogen plays a crucial role in the formation of a highly porous hydrogen-rich layer, within which the nucleation of crystallites takes place. This effect of hydrogen has also been observed during its implantation in crystalline silicon, where it forms hydrogen platelets and is the origin of the Smart-Cut $\AA$ process ${ }^{21}$

\section{SUMMARY AND CONCLUSION}

The nucleation and growth of microcrystalline silicon on an amorphous silicon substrate have been investigated in situ by ellipsometry and dark conductivity measurements. The three phases of incubation, nucleation and growth have been clearly identified from the analysis of the ellipsometry data by BEMA model and the evolution of the dark conductivity of the film. Moreover, these techniques suggest the formation of a highly porous layer and the nucleation of crystallites within this layer. These results have been confirmed by cross sectional high-resolution transmission electron microscopy measurements, which clearly show the presence of a low density layer and its evolution during deposition. Finally, the thicknesses deduced from the HRTEM photographs are in good agreement with those predicted by the BEMA model.

\footnotetext{
${ }^{1}$ Veprek

${ }^{2}$ I.Solomon, B.Drévillon, H.Shirai, and N.Layadi, J.Non-Cryst.Solids, 164-166 ,989,(1993)

${ }^{3}$ C.C.Tsai, G.B.Anderson, R.Thompson, and B.Wacker, J.Non-Cryst.Solids 114, 151 (1989)

${ }^{4}$ K.Nomoto, Y.Urano, J.L.Guizot,G.Ganguly, and A.Matsuda, Jpn.J.Appl.Phys. 1 29, L1372 (1990)

${ }^{5}$ N.Shibata, K.Fukada, H.Ohtoshi, J.Hanna, S.Oda, and I.Shimizu, Mater.Res.Soc.Symp.Proc., 95, 225,(1987)

${ }^{6}$ Y.H.Yang, M.Katiyar, G.F.Feng, N.Maley, and K.R.Abelson, Appl.Phys.Lett. 65, 1789 (1994)

${ }^{7}$ N. Layadi Phys. Rev

${ }^{8}$ C. Godet, N. Layadi, and P. Roca i Cabarrocas: "Role of mobile hydrogen in the amorphous silicon recrystallization". Appl. Phys. Lett. 66 (1995) 3146.

${ }^{9}$ P. Roca i Cabarrocas, N. Layadi, T. Heitz, B. Drévillon, and I. Solomon: "Substrate selectivity in the formation of microcrystalline silicon: Mechanisms and technological consequences". Appl. Phys. Lett. 66 (1995) 3609.
}

\footnotetext{
${ }^{10}$ P. Roca i Cabarrocas, S. Hamma, A. Hadjadj, J. Bertomeu, and J. Andreu: "New features of the layer-by-layer deposition of microcrystalline silicon films revealed by spectroscopic ellipsometry and high resolution transmission electron microscopy". Appl. Phys. Lett. 69 (1996) 529.

${ }^{11}$ P. Roca i Cabarrocas and S. Hamma: "Microcrystalline silicon growth on a-Si:H: Effects of hydrogen". Thin Solid Films 337 (1999) 23

${ }^{12}$ N.Layadi, P.Roca i Cabarrocas, B.Drévillon, and I.Solomon, Phys.Rev.B 51, 5136, (1995)

${ }^{13}$ P.Roca i Cabarocas, N.Layadi, T.Heitz, and B.Drevillon, Appl.Phys.Lett. 66, 3609 (1995)

${ }^{14}$ P.Roca i Cabarrocas,S.Hamma, A.Hadjadj, J.Bertomeu, and J.Andreu, Appl.Phys.Lett. 69, 529 (1996)

${ }^{15}$ N.Layadi, P.Roca i Cabarrocas, J.Huc, J.Y.Parey, and B.Drévillon, Solid State Phenom. 37-38, 281 (1994)

${ }^{16}$ S.Hamma and P.Roca i Cabarrocas, J.ApplPhys., 81 (11), 1 June1997
} 
${ }^{17}$ D.A.G.Bruggeman, Ann.Phys. (Leipzig) 24, 636 (1935)

${ }^{18}$ D.E.Aspnes and A.A.Studna, Phys.Rev. B 27, 1(1993)

${ }^{19}$ G.E.Jellison, Jr,M.F.Chisholm, and S.M.F.Gorbatkin, Appl.Phys.Lett. 62, 3348 (1993)

${ }^{20}=11$

${ }^{21}$ M.Bruel, MRS Bulletin, December 1998,35

Table I. Deposition conditions used in the layer-by-layer process

\begin{tabular}{|l|l|l|}
\hline $\begin{array}{l}\text { Substrate } \\
\text { Temperature } \mathbf{2 0 0}^{\circ} \mathbf{C}\end{array}$ & Deposition & Hydrogen plasma treatment \\
\hline Gas flow & $\begin{array}{l}1 \mathrm{sccm} \mathrm{SiH}_{4} \\
150 \mathrm{sccm} \mathrm{H}_{2}\end{array}$ & $150 \mathrm{sccm} \mathrm{H}_{2}$ \\
\hline Total pressure & $850 \mathrm{mTorr}$ & $750 \mathrm{mTorr}$ \\
\hline Time & $\mathrm{T}_{\mathrm{Si}}=20 \mathrm{~s}$ & $\mathrm{~T}_{\mathrm{H} 2}=120 \mathrm{~s}$ \\
\hline RF power & $10 \mathrm{~W}$ & $30 \mathrm{~W}$ \\
\hline
\end{tabular}




\section{Figure captions}

Fig. 1. Evolution of the imaginary part of the pseudodielectric function. In the first 9 cycles (a) $<\varepsilon_{2}>$ decreases while afterwards (b) $<\varepsilon_{2}>$ shows an opposite trend.

Fig. 2. Results of the optical model used to fit the ellipsometry spectra.

Fig. 3. Evolution of dark conductivity during the LBL cycles.

Fig. 4. a and b show high resolution electron microscopy photographs for samples 80526A and 80526B. Note the increase on the grain size and the reduction of the low density regions form eight to fifteen cycles. 

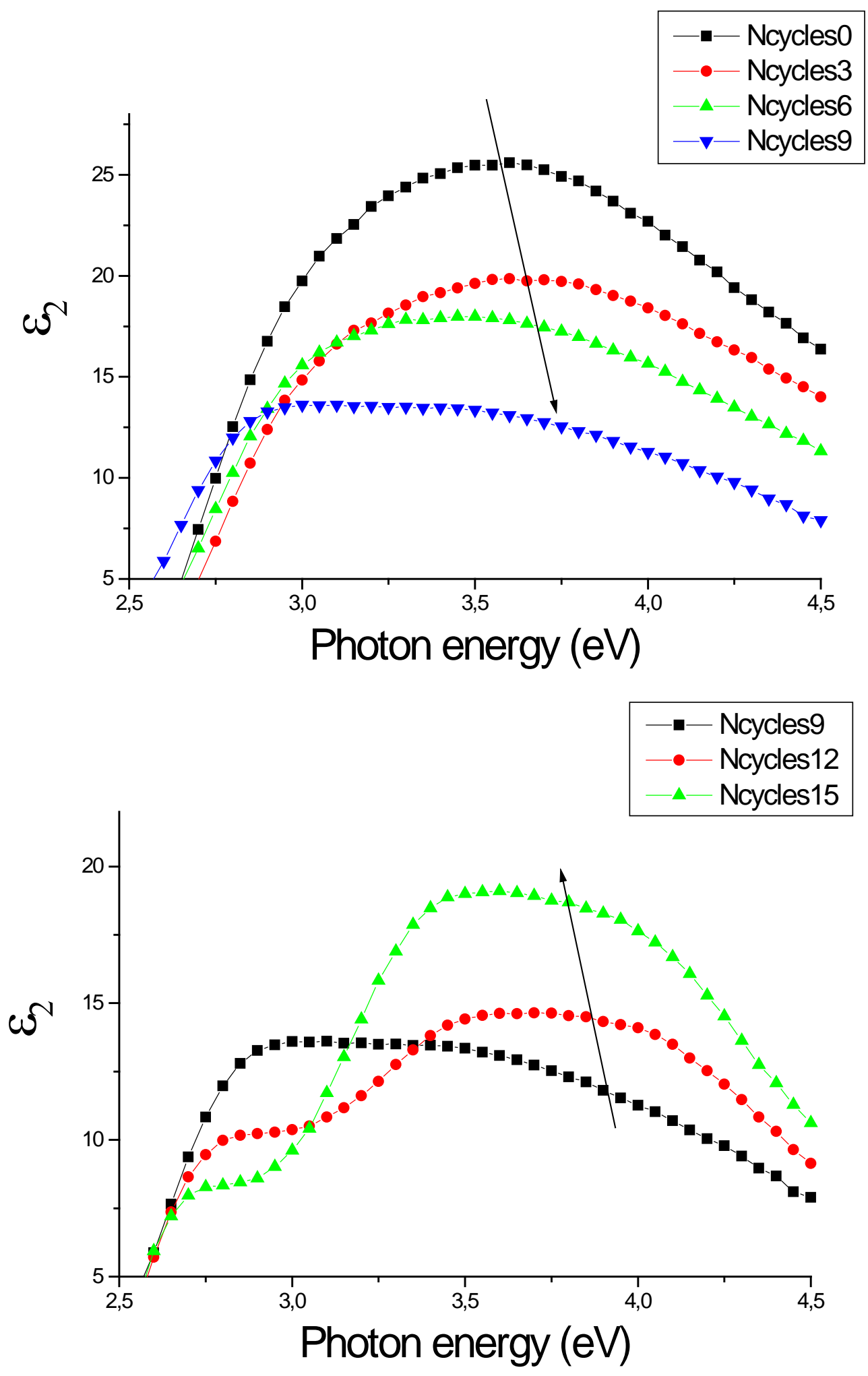
0 Cycles : the substrate

\begin{tabular}{|c|c|c|c|}
\hline$\%$ a-Si :H & $\%$ void & $\%$ crist. & Depth (A) \\
\hline 31 & 69 & I & 15 \\
\hline 98 & 2 & I & 574 \\
\hline & Corning & Glass & Total : 589A \\
\hline
\end{tabular}

\begin{tabular}{|c|c|c|c|}
\hline$\%$ a-Si :H & $\%$ void & $\%$ crist. & Depth (A) \\
\hline 35 & 65 & I & 29 \\
\hline 85 & 15 & 1 & 271 \\
\hline 92 & 8 & 1 & 313 \\
\hline \multicolumn{2}{|r|}{ Corning } & Glass & Total : 613 \\
\hline
\end{tabular}

nucleation and formation of the first crystallites

9 Cycles :

\begin{tabular}{|c|c|c|c|}
\hline$\%$ a-Si :H & $\%$ void & $\%$ crist. & Depth (A) \\
\hline 8 & 52 & 40 & 79 \\
\hline 85 & 15 & 1 & 355 \\
\hline 92 & 8 & 1 & 233 \\
\hline \multicolumn{2}{|r|}{ Corning } & Glass & Total : 667 \\
\hline
\end{tabular}

$$
\text { growth of the crystallites }
$$

\begin{tabular}{|c|c|c|c|}
\hline$\%$ a-Si :H & $\%$ void & $\%$ crist. & Depth (A) \\
\hline 1 & 53 & 47 & 35 \\
\hline 39 & 16 & 45 & 216 \\
\hline 78 & 22 & l & 50 \\
\hline 95 & 5 & I & 481 \\
\hline \multicolumn{2}{|r|}{ Corning } & Glass & Total : 732 \\
\hline
\end{tabular}




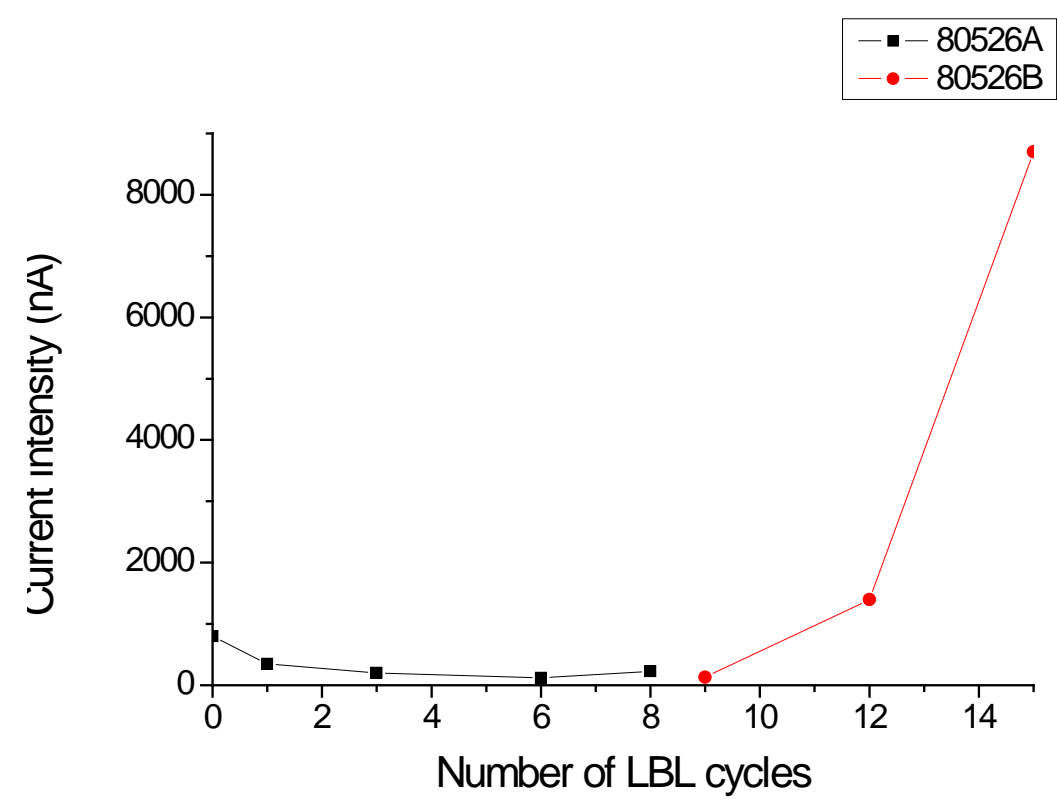


Sample 80526A: 8 cycles

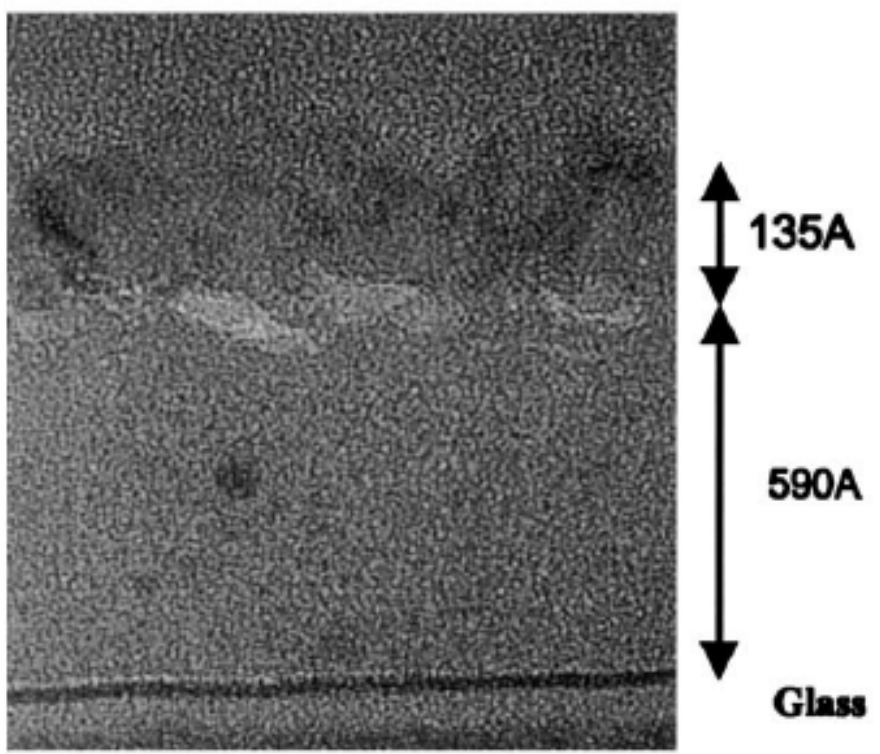

Sample 80526B: 15 cycles
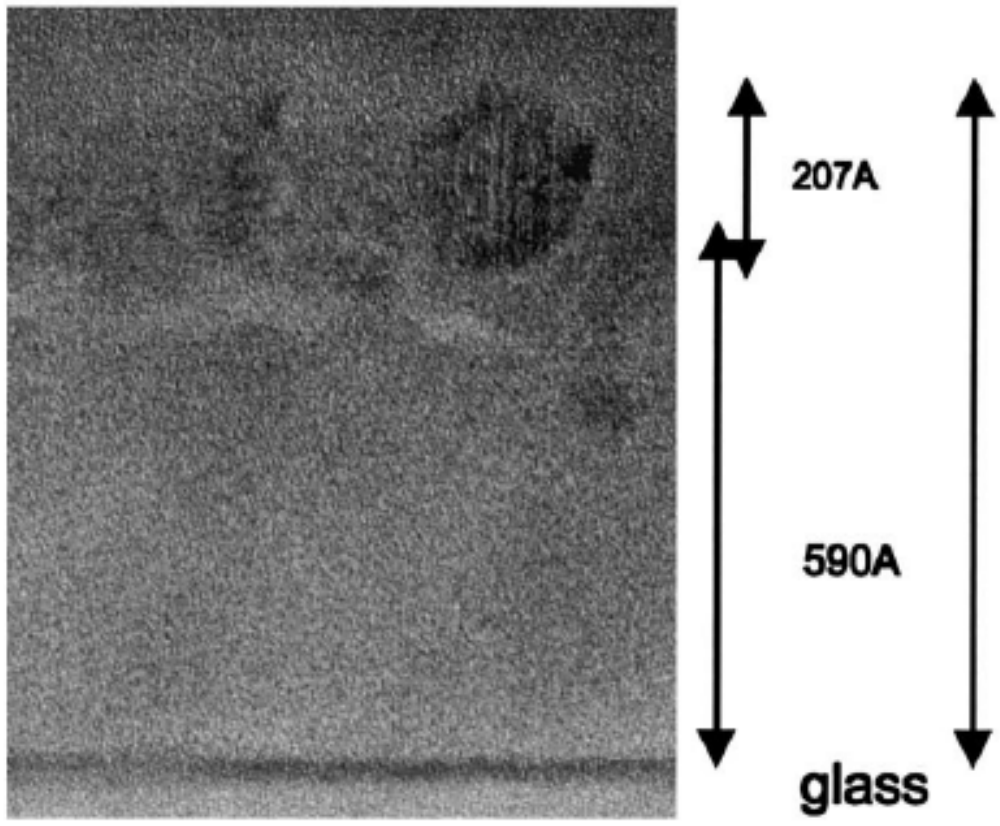

756A 